\title{
Comment on Lockwood and Davis, "On the longitudinal extent of magnetopause reconnection pulses"
}

\author{
W. J. Heikkila \\ Center for Space Sciences, University of Texas at Dallas, Richardson, e-mail: heikkila@utdallas.edu \\ Received: 28 February 1997 / Revised: 26 September 1997/ Accepted: 23 March 1998
}

\begin{abstract}
Lockwood and Davis (1996) present a concise description of magnetopause reconnection pulses, with the claimed support of three types of observations: (1) flux transfer events (FTE), (2) poleward-moving auroral forms on the dayside, and (3) steps in cusp ion dispersion characteristics. However, there are a number of errors and misconceptions in the paper that make their conclusions untenable. They do not properly take account of the fact that the relevant processes operate in the presence of a plasma. They fail to notice that the source of energy (a dynamo with $\mathbf{E} \cdot \mathbf{J}<0$ ) must be close to the region of dissipation (the electrical load with $\mathbf{E} \cdot \mathbf{J}>0$ ) in transient phenomena, since energy (or information) cannot travel faster than the group velocity of waves in the medium (here the Alfvén velocity $V_{A}$ ). In short, Lockwood and Davis use the wrong contour in their attempt to evaluate the electromotive force (emf). This criticism goes beyond their article: a dynamo is not included in the usual definition of reconnection, only the reconnection load. Without an explicit source of energy in the assumed model, the idea of magnetic reconnection is improperly posed. Recent research has carried out a superposed epoch analysis of conditions near the dayside magnetopause and has found the dynamo and the load, both within the magnetopause current sheet. Since the magnetopause current is from dawn to dusk, the sign of $\mathbf{E} \cdot \mathbf{J}$ reflects the sign of the electric field. The electric field reverses, within the magnetopause; this can be discovered by an application of Lenz's law using the concept of erosion of the magnetopause. The net result is plasma transfer across the magnetopause to feed the low latitude boundary layer, at least partly on closed field lines, and viscous interaction as the mechanism by which solar wind plasma couples to the magnetosphere.
\end{abstract}

\section{Introduction}

Lockwood and Davis (1996; hereafter denoted by LD) present a concise description of magnetopause reconnection pulses, with the claimed support of three types of observations: (1) flux transfer events (FTE), a concept introduced by Russell and Elphic (1978); (2) polewardmoving auroral forms on the dayside, first investigated in detail by Sandholt et al. (1986); and (3) steps in cusp ion dispersion characteristics, which are common in low altitude satellite observations of the dayside cusp (e.g. Woch and Lundin, 1992). However, there are a number of errors and misconceptions in the paper that make their conclusions untenable. They do not properly take account of the fact that the relevant processes operate in the presence of a plasma. Furthermore, it is difficult to check causality of their envisioned process.

\section{Pulsed reconnection}

LD begin by using the definition of pulsed reconnection: "By definition, a pulse of reconnection during southward IMF conditions will produce a patch of newly opened flux in the ionosphere and a patch of rotational discontinuity on the magnetopause. Both these are threaded by the newly opened field lines ...." I will look closely at the various parts of this definition in the following.

\section{Steady state reconnection}

Figure 1a is from their article (first of three parts); this shows the usual model for steady state reconnection, usually defined in two dimensions (e.g. Sonnerup, 1985). If an electric field is present along the $\mathrm{X}$-line (denoted by $A B$ ), then reconnection is defined to occur (Sonnerup, 1985). It should be noted that the conditions for reconnection to occur are not known (LD p. 865; Semenov et al., 1992); instead, the reconnection electric 

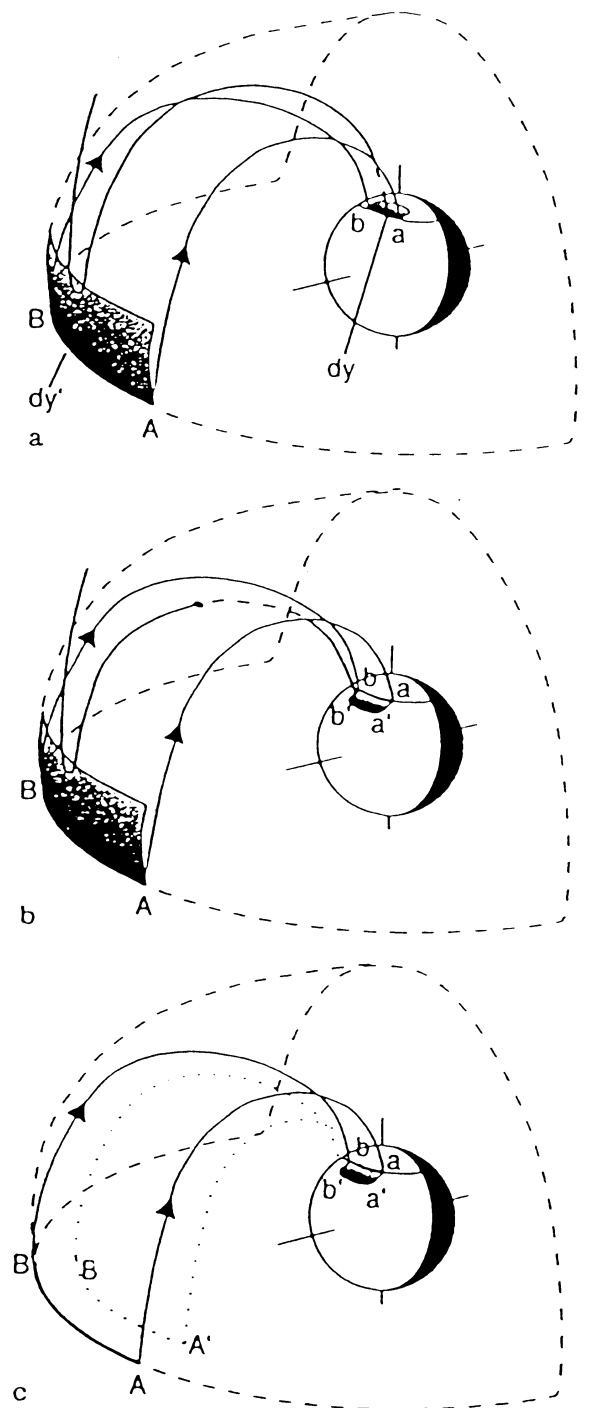

Fig. 1. The possible effects of a reconnection pulse at a magnetopause $\mathrm{X}$-line $A B$, which maps to an ionospheric merging gap $a b$ (after Lockwood and Davis, 1996). a This is the steady-state case where $a b$ and $A B$ are static in the Earth's frame, and there is no change in the magnetic field in the dayside magnetosphere. In this case, the newly opened flux (like the one shown which thread the magnetopause and the ionosphere in the shaded regions) do not thread the Faraday loop $A B b a$ and poleward flow is excited in the ionosphere in the Earth's frame by the reconnection rate. In $\mathbf{b} A B$ is static but $a b$ migrates to $a^{\prime} b^{\prime}$ such that the newly field lines thread the Faraday loop $A B b a$ to the extent that no flow is excited in the ionosphere in the Earth's frame. In $\mathbf{c}$ the $\mathrm{X}$-line $A B$ erodes Earthward to $A^{\prime} B^{\prime}$ and $a b$ moves equatorward to $a^{\prime} b^{\prime}$, again exciting no ionospheric flow in the Earth's frame

field is prescribed as an input parameter. A finite resistivity, the so-called anomalous resistivity (Coroniti, 1985), is usually assumed leading to dissipation along the $\mathrm{X}$-line. Thus we have $\mathbf{E} \cdot \mathbf{J}>0$, called a load in electrical engineering (like a toaster). As stated by Cowley (1980), the plasma gains energy at the expense of the electromagnetic field (consistent with the reconnection hypothesis). Likewise, along $a b$ : the ionosphere will also act as an electrical load, with further dissipation (assuming a small electric field parallel to the magnetic field lines, $E_{\|} \approx 0$ ). Thus we have two loads connected by infinite conductors.

However, there is no source of energy in the assumed model, no dynamo with $\mathbf{E} \cdot \mathbf{J}<0$ (like a battery). In a plasma dynamo the plasma loses energy, supplying it to the electromagnetic field. In the reconnection model (Sonnerup, 1985) the electric circuit is incomplete, unphysical. The situation can be realistic only if the combination is fed by an external dynamo (as in driven reconnection). The ionosphere becomes an electrical shunt, in parallel with the dayside magnetopause load if steady state reconnection (as defined) is going on. It is a matter of cause and effect (Ramo and Whinnery, 1953). Unfortunately, most discussions of reconnection do not even mention this dynamo.

Whenever we have an electrical load (the reconnection load) we must also find a dynamo somewhere in the continuation current; the current must be closed in the system under study for causal reasons. The magnetopause current, in three-dimensions, is closed (solenoidal), forming loops about the dayside cusps (Cowley, 1980). The dynamo is thought to be situated over the lobes of the magnetotail (Cowley, 1980) where the current is from dusk to dawn in the presence of a dawndusk electric field for a southward interplanetary magnetic field, consequently with $\mathbf{E} \cdot \mathbf{J}<0$.

We can proceed more formally. In the steady state we can use the electrostatic potential to describe the electric field; we can readily derive the following relation (see Heikkila, 1997, 1998):

$$
\begin{aligned}
\iiint_{\text {vol }} \mathbf{E} \cdot \mathbf{J d} \tau & =-\oint \oint_{\text {suff }} \frac{\mathbf{E} \times \mathbf{B}}{\mu_{0}} \cdot \mathrm{d} \mathbf{S} \\
& =-\oint \oint_{\text {surf }} \phi \mathbf{J} \cdot \mathrm{d} \mathbf{S}
\end{aligned}
$$

where $\phi$ is the electrostatic potential. Thus, there are two viewpoints for the energy transfer. One is the traditional one, the divergence of the Poynting flux. But, whenever we see a net Poynting flux entering some region we must also have a current in that region describing dissipation with $\mathbf{E} \cdot \mathbf{J}>0$. Exactly the opposite happens when there is a net flow of Poynting flux out of some region; then we must have a current with $\mathbf{E} \cdot \mathbf{J}<0$ characteristic of a dynamo. The cause (the source of energy) is a dynamo, showing just where the plasma loses energy, while the effect (the electrical load) indicates dissipation with particles gaining energy (which could be either thermal energy or a directed beam; the Poynting theorem is silent on that point).

\section{The weakness of a 2-D model}

One of the advantages of using a circuit approach to the energy problem is that the complete circuit must be used. Furthermore, the question of dimensionality in physical space is irrelevant to the electric circuit that needs to be used.

Of course it should be obvious that a two-dimensional model must be used with care in attempting to use 
it to explain effects in three dimensions. We can use Poynting's theorem to elucidate the exchange of energy between the electromagnetic field and the kinetic energy of particles [not commonly done in magnetohydrodynamics (MHD)]:

$$
\begin{aligned}
& \iiint_{\mathrm{vol}} \mathbf{E} \cdot \mathbf{J} \mathrm{d} \tau=-\oint \oint_{\text {surf }} \frac{\mathbf{E} \times \mathbf{B}}{\mu_{0}} \cdot \mathrm{d} \mathbf{S} \\
& -\iiint_{\mathrm{vol}}\left(\varepsilon_{0} \mathbf{E} \cdot \frac{\partial \mathbf{E}}{\partial t}+\frac{\mathbf{B}}{\mu_{0}} \cdot \frac{\partial \mathbf{B}}{\partial t}\right) \mathrm{d} \tau
\end{aligned}
$$

The possible sources for the energy integral on the lefthand side (for example, the reconnection load) are described by the terms on the right. Steady state reconnection theories consider only the first term, which corresponds to a flow of energy to the reconnection load from an external source. Any internal magnetospheric source must be described by a volume integral, and both those integrands vanish unless the electromagnetic field is time-dependent. These latter two terms specify the rate of increase or decrease of stored electric and magnetic energies, respectively (depend on polarization and induction electric fields). It is difficult, if not impossible, to represent any 3-D time-dependent process with a steady-state two-D model. In particular, we must be careful in extending the ideas on steady-state reconnection to the time-dependent case.

\section{The definition of pulsed reconnection}

In trying to reach the correct mathematical formulation LD do correctly base their logic on Faraday's law, in fact the more meaningful integral form in their Eq. (1) expressing the electromotive force (emf):

$$
\begin{aligned}
-\oint_{A B b a} E \cdot \mathrm{d} l & =E_{t} \mathrm{~d} y^{\prime}-E_{i} \mathrm{~d} y \\
& =\mathrm{d} / \mathrm{d} t\left(-\int_{A B b a} B \cdot \mathrm{d} a\right) \\
& =\mathrm{d} F / \mathrm{d} t
\end{aligned}
$$

where $E_{t}$ is the electric field along $A B$ and $E_{i}$ is the electric field along the merging gap, both being measured in the Earth's frame of reference. $F$ is the magnetic flux threading the Faraday loop $A B b a$.

In the steady state illustrated by Fig. 1a the X-line $A B$ and the merging gap $a b$ are both static, and the (assumed) electric field $E_{t} \mathrm{~d} y^{\prime}$ is equal to $E_{i} \mathrm{~d} y$; moreover, $E_{t}$ is equal to the electric field $\xi$ along $A B$ in its own rest frame and therefore it defines the reconnection rate. Magnetic flux is transported poleward, in equal amounts, with $F$ being constant; it should be noted that only electrical loads are considered by LD. Figure 1b, however, shows the results when reconnection is unable to move the ionospheric plasma. LD state that "on short time scales (they say less than $1 \mathrm{~min}$, p. 868) this applies because of the drag produced by collisions of ions with the much more numerous neutral particles"; this implies that the electric field $E_{i}$ is already present. On the other hand, on p. 868 they say that
$E_{i}=0$ but $a b$ now migrates equatorward to $a^{\prime} b^{\prime}$ such that all newly opened flux threads the Faraday loop $A B b a$ (again, only loads). In part (c) the magnetosphere erodes Earthwards such that the X-line migrates from $A B$ to $A^{\prime} B^{\prime}$ with $\xi$ in the magnetopause frame finite while $E_{t}$ in the Earth's frame falls to zero.

\section{Patch of rotational discontinuity on the magnetopause}

LD assume that the reconnection process is necessary to create the rotational discontinuity, it being assumed that the tangential discontinuity is usually present unless "reconnection" is going on. However, it seems likely that a rotational discontinuity will be present over most regions, depending only on the IMF. As a thought experiment, begin with no plasma; there will be a separatrix dividing the magnetic field lines with their respective sources, including field lines connecting the high-latitude magnetic field with the IMF. Then we begin adding plasma, but the current carrying abilities of the plasma at first will not be enough to form a magnetopause, and no "reconnection". Eventually we will get a magnetopause, but I doubt that it will be a tangential discontinuity; a tangential discontinuity must be the exception, just the opposite of that assumed by LD. The result of Phan et al. (1994) in their separation of data into low- and high-shear cases argues strongly for my view.

\section{Patch of newly opened flux in the ionosphere}

LD continue with their version of the temporal development of pulsed reconnection, not noticing that they have made a serious error in their logic. The error they make is the following: energy, or information, cannot travel faster than the group velocity of waves in the medium. Assuming fluid (MHD) theory (as they do), this would be the Alfvén velocity $V_{A}$; information, or energy, cannot propagate super-Alfvénically in the given medium. Since $V_{A}$ is not very high, probably a few hundred kilometers per second, the information may take a few minutes to reach the ionosphere at a distance of some 10 to $15 R_{E}$, say about $2-5 \mathrm{~min}$ (perhaps as low as their $1 \mathrm{~min}$ ). Individual particles of higher energy can of course preceed the plasma response as a precursor of things to come, but they cannot change the ionospheric medium by definition of the group velocity. The physical processes at work at the magnetopause cannot wait for the ionospheric response; erosion of the magnetopause does happen quickly. Phan and Paschmann (1996) found an average normal component of plasma velocity of $20 \mathrm{~km} / \mathrm{s}$ for such erosion. With a thickness of the magnetopause current sheet of about $500 \mathrm{~km}$ (Berchem and Russell, 1982), we find a typical interaction time of 25 s. If we use the tangential magnetosheath flow, which is super-Alfvénic toward the dawn and dusk flanks at about $300-400 \mathrm{~km} / \mathrm{s}$, a region $1 R_{E}$ wide is swept out in 15-20s. These estimates are about an order of magnitude less than the communication time to the iono- 
sphere. In fact, the first impulse occurs in even less time than that, at the first contact of the new solar wind plasma with the magnetopause.

The conclusion is that the plasma physical processes involved in erosion of the magnetopause, the plasma physics associated with solar wind-magnetospheric interaction, must all happen within a very localized region in space near the magnetopause, say (to be extravagant) $1 R_{E}$ in radius (Heikkila, 1994). The different interaction regimes in the sizeable magnetopause region cannot communicate with the ionosphere (or with each other) for lack of time (Heikkila, 1986, 1994, 1997, 1998). Speaking in causal terms, the cause (the source of energy) must be close to the effect (dissipation). In electrical terms, the dynamo (with $\mathbf{E} \cdot \mathbf{J}<0$ ) must be close to its electrical load (with $\mathbf{E} \cdot \mathbf{J}>0$ ). A dynamo over the lobe magnetopause is entirely out of the question.

It is not clear where LD wish to put their dynamo. Their method of evaluating the emf driving "reconnection", taking the line integral over a path extending all the way to the ionosphere, is arguably erroneous.

\section{Perturbation of the magnetopause current}

I submit that the proper way to attack the problem of erosion of the magnetopause is shown by Fig. 2. The undisturbed magnetopause current is shown at the left, at time $t_{0}$. After erosion has begun the current is deformed as shown at a later time $t_{1}=t_{0}+\Delta t$. Such a deformation can be represented by adding a current perturbation $\delta \mathbf{J}$ to the undisturbed current; Lenz's law can now be applied to the perturbation current, all locally within the magnetopause. An inductive electric
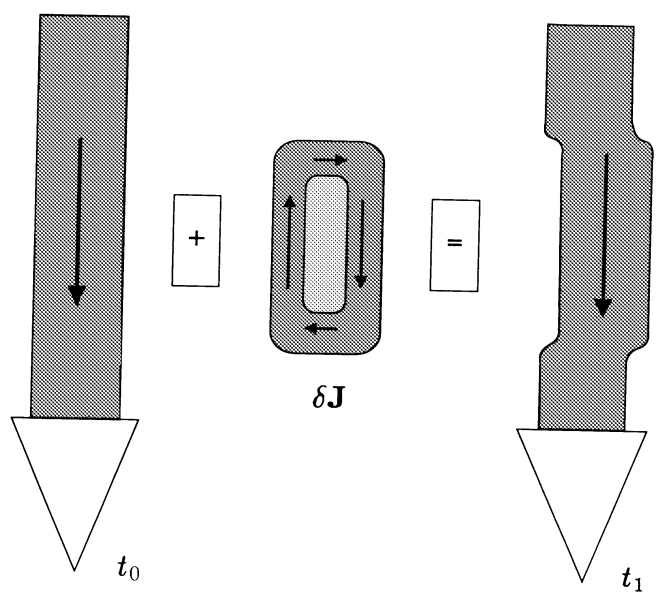

Fig. 2. The inward meander of the current sheet at time $t_{1}$ associated with erosion of the magnetopause is equivalent to the former current at time $t_{0}$ plus a perturbation current loop, with $\operatorname{div} \delta \mathbf{J}=0$. A clockwise perturbation is needed to create more open flux; by Lenz's law, an induction electric field in the counter-clockwise sense will exist opposing the current perturbation everywhere, with an electromotive force $\varepsilon=-\mathrm{d} \Phi^{M} / \mathrm{d} t$ evaluated by a taking a line integral around the current perturbation field $\mathbf{E}^{\text {ind }}=-\partial \mathbf{A} / \partial t$ is the result where $\mathbf{A}$ is the vector potential.

The electric field due to induction will try to drive charges in the plasma; the controlling factor in its ability to do that is the magnetic field. Away from the magnetopause itself the magnetic field is almost tangential to the magnetopause because of the generally small normal component of the magnetic field $B_{n}$ : thus we have the usual $\mathbf{E} \times \mathbf{B}$ drift and no polarization due to the small value of the Pedersen conductivity in a collisionless plasma. However, the situation is completely different within the current layer. In particular, the key factor is a finite $B_{n}$ (if present), associated with a rotational discontinuity. One aspect is that the plasma can simply flow along $B_{n}$. Another even more important aspect is that the normal component $E_{n}^{\text {ind }}$ now becomes $E_{\|}^{\text {ind }}$; this rules heavily against using fluid theory for this application. Note again that $E_{\|}^{\text {ind }}$ is not affected by the Lorentz transformation, and that the direct conductivity is very large.

The plasma can easily polarize along $B_{n}$ (if present) so as try to cancel (or at least reduce) $E_{\|}^{\text {ind }}$. However, an electrostatic field can have no effect on the electromotive force of the inductive field because its curl vanishes: any reduction in the net $E_{\|}$in any arbitrary closed contour must involve enhancement of the perpendicular component $E_{\perp}$ at least somewhere along that contour, otherwise the curl (or emf) would be affected. These charges will create an electrostatic field across the perturbation, reversing on the two sides of the magnetopause current because of the quadrupole nature of the charge distribution.

Now we see the reason for the reconnection electric field; it is an electrostatic field caused by the plasma, reacting to the induction field due to the erosion. However, it is accompanied by an oppositely directed electric field where the current is increasing, again because of the quadrupole nature of the charge distribution. It is the field that is associated with the dynamo that Phan and Paschmann (1996) have found in their analysis of $T_{e}$ (for more details see Heikkila, 1997, 1998).

This is very different from that assumed by LD in their Fig. 1. They assumed, without specifying any mechanism (other than the dubious one of anomalous resistivity), a unidirectional electric field at the magnetopause, one with curl $\mathbf{E}=0$, this despite the fact that they were discussing time-dependence, Faraday's law notwithstanding.

\section{The claimed support}

\section{Flux transfer events}

The above description of the electric profile through the magnetopause current, which is relevant to erosion, has serious implications for frozen-in flow and the very concept of flux transfer events (FTEs). Since both the magnetic field (for a southward IMF), and now the electric field reverse across the magnetopause their 
cross-product will not reverse. The electric drift term $\mathbf{E} \times \mathbf{B}$ will be unidirectional (thus making it easy for the conservation of momentum).

The outcome is that we cannot use the concept of frozen-in magnetic field at the magnetopause, or the concept of FTE. Flux transfer at the magnetopause is unphysical. The plasma goes tailward, even to closed field lines, not poleward to open field lines. We cannot use MHD to discover this behaviour.

\section{Poleward moving auroral forms}

The question of poleward moving auroral forms on the dayside is an intriguing one. It is almost certainly caused by solar wind plasma interacting with the magnetopause, or with the boundary layer just inside. It cannot be discussed with the concept of frozen-field convection (as concluded in the previous section). The solar wind plasma in the LLBL or mantle is still convecting tailward but now at least partly on closed field lines. More than likely, the poleward moving auroral forms are due to the drifting particles in the LLBL, on closed field lines, flowing anti-sunward in accordance with Newton's laws.

\section{Cusp ion dispersion}

Multiple injections are easily understandable with the impulsive penetration concept. The driven response is determined by the magnetosheath flow. Once the plasma gets into the boundary layer there are various processes for the loss of momentum of the plasma: (1) the need to accelerate the magnetospheric and ionospheric plasma (Lundin, 1988), and (2) field aligned currents, which leads to an efficient braking mechanism transferring momentum to ionospheric plasma (Lemaire and Roth, 1978). Thus, it is reasonable to expect that the tailward velocity in the LLBL would be lower than in the adjacent magnetosheath; it is also observed to be lower, at about $200 \mathrm{~km} / \mathrm{s}$. Thus the observed plasma in the LLBL proceeds at a slower velocity than in the magnetosheath. Now the possibility of a new injection occurs while the plasma from the previous injection is still there, still drifting tailward. The observations support impulsive transfer of plasma, not magnetic flux.

\section{Conclusion}

The main point of this comment is that the rate of information flow, such as the requirements for the electric field for a change in plasma convection, and also for the energy transfer, is limited by the group velocity of waves in the medium. This is a necessary condition for any physical theory. Faced with this condition, it is certain that the situation which Fig. 1 of LD is meant to convey is unphysical. In short, LD use the wrong contour in evaluating the emf. What happens in the ionosphere does not matter to the first intrusion of magnetosheath plasma at the magnetopause, the first push. All the action must happen in a very limited region in space, as in Fig. 2, where the electrical circuit is provided by the current perturbation $\delta \mathbf{J}$. Here, both the dynamo and the electric load are in the same circuit close to each other. This has now been verified by Phan and Paschmann (1996). This must be so for a proper discussion of cause and effect.

Acknowledgement. Funding was provided by the National Science Foundation under Grant ATM-960117.

\section{References}

Berchem, J., and C. T. Russell, The thickness of the magnetopause current layer: ISEE 1 and 2 observations, J. Geophys. Res., 87, 2108, 1982.

Coroniti, F. V., Space plasma turbulent dissipation: reality or myth?, Space Sci. Rev., 42, 399, 1985.

Cowley, S. W. H., Plasma populations in a simple open model magnetosphere, Space Sci. Rev., 26, 217, 1980.

Heikkila, W. J., Transport of plasma across the magnetopause, in Solar Wind-Magnetosphere Coupling, Eds.: Y. Kamide, and J. A. Slavin, D. Reidel, Tokyo, p. 337, 1986.

Heikkila, W. J., Another look at flux transfer events, in Physical signatures of magnetospheric boundary layer processes, Eds.: J. A. Holtet, and Alv Egeland, Kluwer Academic Publ., Dordrecht, The Netherlands 1994.

Heikkila, W. J., Interpretation of Recent APMTE Data at the Magnetopause, J. Geophys. Res., 102, 2115, 1997.

Heikkila, W. J., Cause and effect at the magnetopause, Space Sci. Rev., 83, 373, 1998.

Lemaire, J., and M. Roth, Penetration of solar wind plasma elements into the magnetosphere, J. Atmosph. Terr. Phys., 40, 331, 1978.

Lockwood, M., and C. J. Davis, On the longitudinal extent of magnetopause reconnection pulses, Ann. Geophysicae, 14, 865, 1996.

Lundin, R., On the magnetospheric boundary layer and solar wind energy transfer into the magnetosphere, Space Sci. Rev., 48, 263, 1988.

Phan, T.-D., and Götz Paschmann, The low-latitude dayside magnetopause and boundary layer for high magnetic shear: 1 . Structure and motion, J. Geophys. Res., 101, 7801, 1996.

Phan, T.-D., G. Paschmann, W. Baumjohann, N. Sckopke, and H. Lühr, The magnetosheath region adjacent to the dayside magnetopause: AMPTE/IRM observations, J. Geophys. Res., 99, 121, 1994.

Ramo, S. and J. Whinnery, Fields and Waves in Modern Radio, Wiley, New York 1953.

Russell, C. T., and R. C. Elphic, Initial ISEE magnetometer results: Magnetopause observations, Space Sci. Rev., 22, 681, 1978.

Sandholt, P. E., C. S. Deehr, A. Egeland, B. Lybekk, R. Viereck, and G. J. Romick, Signatures in the dayside aurora of plasma transfer from the magnetosheath, J. Geophys. Res., 91, 10063, 1986.

Semenov, V. S., I. V. Kubyshkin, V. V. Lebedeva, M. V. Sidneva, H. K. Biernat, M. F. Heyn, B. P. Besser, and R. P. Rijnbeek, J. Geophys. Res., 97, 4251, 1992.

Sonnerup, B. U. Ö., Magnetic field reconnection in cosmic plasmas, in Unstable Current Systems and Plasma Instabilities in Astrophysics, Ed.: M. R. Kundu, and G. D. Holman, Reidel, Norwell, Mass., 1985.

Woch, J., and R. Lundin, Signatures of transient boundary layer processes observed with Viking, J. Geophys. Res., 97, 1431, 1992. 